\title{
BMJ Open Herbal medicines for cancer cachexia: protocol for a systematic review
}

\author{
Bongki Park, Ji Hee Jun, Jeeyoun Jung, Sooseong You, Myeong Soo Lee
}

To cite: Park B, Jun JH, Jung J, et al. Herbal medicines for cancer cachexia: protocol for a systematic review. BMJ Open 2014;4:e005016.

doi:10.1136/bmjopen-2014005016

- Prepublication history and additional material is available. To view please visit the journal (http://dx.doi.org/ 10.1136/bmjopen-2014005016).

Received 7 February 2014 Revised 10 May 2014 Accepted 13 May 2014
CrossMark

Medical Research Division, Korea Institute of Oriental Medicine, Daejeon, South Korea

Correspondence to Dr Myeong Soo Lee; drmslee@gmail.com

\section{ABSTRACT}

Introduction: To assess the efficacy of herbal medicines as a treatment of cancer cachexia.

Methods and analysis: We will search the following 13 electronic databases from their inception. MEDLINE (PubMed), the Cochrane Central Register of Controlled Trials (CENTRAL), EMBASE, Allied and Complementary Medicine Database (AMED), China National Knowledge Infrastructure (CNKI), Wanfang, Journal Integration Platform (VIP) and six Korean Medical Databases (KoreaMed, the Korean Traditional knowledge Portal, OASIS, DBPIA, the Research Information Service System and the Korean Studies Information Service System) without restrictions on time or language. The data will be extracted independently by two authors using predefined criteria. Disagreements will be resolved by discussion between the authors. The risk of bias will be assessed using the Cochrane risk of bias tool.

Dissemination: The review will be published in a journal. The review will also be disseminated electronically and in print. An update of the review will be conducted to inform and guide healthcare practice and policy.

Trial registration number: PROSPERO 2013: CRD42013006612.

\section{INTRODUCTION}

\section{Description of the condition}

Cancer cachexia is a common syndrome among patients with cancer, especially advanced cancer. More than $70-80 \%$ of patients with advanced cancer suffer from cachexia. ${ }^{1}$

Generally, cachexia is characterised by loss of weight, muscle atrophy, anorexia and fatigue. The definition of cancer cachexia differs slightly according to the research study, ${ }^{2-5}$ but the recent international consensus has defined cancer cachexia as a multifactorial syndrome characterised by ongoing loss of skeletal muscle mass (with or without loss of fat mass) that cannot be fully reversed by conventional nutritional support and leads to progressive functional impairment. ${ }^{4}$ The pathophysiology of cancer cachexia is a negative energy balance caused by a variable

\section{Strengths and limitations of this study}

- The strength of this review is its extensive, unbiased search of various databases without language restriction.

- The trial screening and data extraction will be conducted independently by two authors.

- The review team consists of experts from each field: traditional Korean medicine (BP and $\mathrm{JJ}$ ), traditional Chinese medicine ( $\mathrm{JHJ}$ ), conventional medicine (SY) and methodology (MSL).

combination of reduced food intake and abnormal metabolism. ${ }^{4}{ }^{6}$ The metabolic mechanisms of adipose mass reduction, loss of skeletal muscle and protein degradation are known to be mediated by proinflammatory cytokines, neuropeptides, hormones, catabolic factors and digestive factors. ${ }^{16-8}$

Cancer cachexia causes numerous clinical problems. It reduces activity or quality of life ${ }^{9}$ and restricts conventional therapy such as chemotherapy. ${ }^{10}$ Above all, cancer cachexia is associated with a poor survival rate. ${ }^{11} 12 \mathrm{In}$ various cancer types, the survival of patients with cachexia is shorter than that of other patients. ${ }^{1}$

\section{Description of the intervention}

There are several agents for managing cancer cachexia. Megestrol acetate, cannabinoids, corticosteroids and ghrelin are known to affect appetite. Eicosapentaenoic acid (EPA), $\beta$-hydroxy- $\beta$-methylbutyrate (HMB), thalidomide, corticosteroids and nonsteroidal anti-inflammatory drugs are known to affect cachectic mediators or signal pathways. ${ }^{1} 6{ }^{13}$ Some drugs, such as megestrol acetate and corticosteroids, exhibit confirmed effectiveness in combating cancer cachexia by randomised controlled trials and systematic reviews. ${ }^{1}$ However, until now, there is no identified standard treatment for cancer cachexia.

Herbal medicines have been used widely to treat diverse diseases for thousands of years. Herbal medicines have been 
developed on the basis of unique theories, especially in East Asia: Yin and Yang, the five elements and visceral manifestation theory. The major principle of treating diseases is reinforcing the healthy qi and eliminating the pathogenic factors. Many herbal medicines are prescribed according to this principle.

Recently, herbal medicines have been used to alleviate the adverse effects of conventional therapies or to improve the quality of life. A survey demonstrated that $75 \%$ of colorectal patients with cancer used at least one type of complementary alternative medicine (CAM) during their lifetime. ${ }^{14}$ Another survey reported that $39 \%$ of patients with breast cancer used herbs or medical herbal teas. ${ }^{15}$

\section{How the intervention might work}

Some herbs, such as ginseng radix, astragali radix or some herbal prescriptions, are known to increase appetite, facilitate physical function and boost immune function. ${ }^{16-20}$ Other herbs, such as coptidis rhizoma, exhibit antiinflammatory functions. ${ }^{21-23}$ Many herbs are associated with anticancer properties including cancer cellcytotoxicity, cell-apoptosis and invasion and metastasis prevention. ${ }^{24-26}$

Many practitioners and researchers have attempted to explore herbal medicines for the treatment of cancer cachexia. The oral administration of Rikkunshito is known to stimulate ghrelin secretion. ${ }^{27}$ One research study reported that coptidis rhizoma might exhibit an anticachectic effect and that berberine, the major component, might prevent cancer-induced cachexia. ${ }^{28}$

\section{Why it is important to do this review}

To the best of our knowledge, no systematic reviews assessing herbal medicines in cancer cachexia have been conducted. There is no identified standard treatment for cancer cachexia, and a comprehensive evaluation of the efficacy and the safety of herbal medicines will inform the recommendation for treatment of cancer cachexia.

\section{OBJECTIVES}

To assess the efficacy of herbal medicines for cancer cachexia.

\section{METHODS}

\section{Criteria for considering studies for this review}

Types of studies

Randomised controlled trials (RCTs) and quasirandomised trials (quasi-RCTs) will be included in this systematic review without restrictions on time or language.

\section{Types of participants}

Participants will include patients with cancer diagnosed by histological or clinical diagnosis who meet the international consensus of cancer cachexia. ${ }^{4}$ The diagnostic criteria for cancer cachexia are as follows:
1. Weight loss $>5 \%$ over the past 6 months (in the absence of simple starvation); or

2. Body mass index (BMI) $<20$ and any degree of weight loss $>2 \%$; or

3. Appendicular skeletal muscle index consistent with sarcopenia (males $<7.26 \mathrm{~kg} / \mathrm{m}$; females $<5.45 \mathrm{~kg} / \mathrm{m}$ ) and any degree of weight loss $>2 \%$.

\section{Types of interventions}

All types of herbal medicines will be included. There is no limitation on the number of herbs, administration methods, dosage or duration of treatment. The comparisons will be either with other therapeutic agents such as megestrol acetate or corticosteroids or with no other treatment.

\section{Types of outcome measures}

Primary outcomes

1. Weight gain

2. Body composition

\section{Secondary outcomes}

1. Improvement in quality of life by means of a validated instrument

2. Increase of appetite

3. Reduction in fatigue

4. Serum levels of inflammatory markers, including erythrocyte sedimentation rate and $\mathrm{C}$ reactive protein as well as tumour necrosis factor- $\alpha$, Interleukin 1 (IL-1), Interleukin 6 and infernon- $\gamma$

5. Survival rate

6. Adverse effects

\section{Search methods for the identification of studies}

\section{Electronic searches}

We will search the following electronic databases regardless of publication date or language:

- MEDLINE (PubMed)

- The Cochrane Central Register of Controlled Trials (CENTRAL)

- EMBASE

- Allied and Complementary Medicine Database (AMED)

- China National Knowledge Infrastructure (CNKI)

- Wanfang Database

- VIP (Journal Integration Platform)

- Six Korean Medical Databases (KoreaMed, the Korean Traditional Knowledge Portal, OASIS, DBPIA, the Research Information Service System and the Korean Studies Information Service System).

\section{Other sources}

We will scan the reference lists of reviews and retrieve articles for additional studies. In addition, we will search the WHO International Clinical Trials Registry Platform (ICTRP) (http://apps.who.int/trialsearch/) and Google scholar (http://scholar.google.co.kr/). 


\section{Search strategy}

We will model participant strategies for databases on the search strategy designed for MEDLINE (PubMed; see online supplementary appendix 1) and CNKI (see online supplementary appendix 2) and modify it for use in the other databases.

\section{Data collection and analysis}

\section{Selection of studies}

Two review authors (BP and $\mathrm{JHJ}$ ) will independently assess the titles and abstracts of articles searched by electronic databases and determine their eligibility for inclusion. Hard copies of the relevant articles will be retrieved. Disagreements will be resolved by discussion, if necessary, by the arbiter (MSL).

\section{Data extraction and management}

Two review authors (BP and JHJ) will read all the articles and independently extract the data using a standard data extraction form. The form includes methodology, participants, interventions, duration of treatment, outcomes and conclusions. Consensus will be reached by discussion in the case of discrepancy. When disagreements are not resolved by discussion, they will be arbitrated by another author (MSL).

\section{Assessment of the risk of bias in the included studies}

Three authors (BP, SY and JJ) will assess the risk of bias using the Cochrane tool of risk of bias (V.5.1.0) ${ }^{29}$ The following items will be assessed: random sequence generation (selection bias), allocation concealment (selection bias), blinding (performance bias and detection bias), incomplete outcome data (attrition bias), selective outcome reporting (reporting bias) and other bias. The evaluated domains will be assessed as Yes, No or Unclear according to the criteria. We will resolve any disagreements by discussion or arbitration (MSL).

\section{Measurement of the treatment effect}

We will use the mean difference (MD) with $95 \%$ CIs for continuous outcomes or risk ratios (RR) or odds ratios (OR) with 95\% CI for binary outcomes. If the event rate is lower than $1 \%$, Peto OR with $95 \%$ CI will be used. When the event rate is lower than $20 \%$, OR with $95 \%$ CI will be used. If the event rate is more than $20 \%$, $\mathrm{RR}$ with 95\% CI will be used. In the case of use of different measurement scales, standardised mean difference (SMD) analysis with 95\% CI will be performed.

\section{Units of analysis issues}

We will include data from parallel-group studies for the meta-analysis. If we include cross-over trials, only the first treatment period data will be analysed. When the trial has more than one control group, the unit of analysis will be applied to each group.

\section{Dealing with missing data}

We will try to contact the corresponding authors by e-mail if there are any missing or insufficient data from the trial as much as possible. The intent-to-treat (ITT) principle will be applied for statistical analysis. The individual patient data will be sought from the original source or from the published trial reports when the individual patient data are unavailable.

\section{Assessment of heterogeneity}

We will use the random effects model for the meta-analysis. Heterogeneity will be assessed by inspecting the forest plots. In addition, heterogeneity will be tested by the $\mathrm{I}^{2}$ test for quantifying inconsistencies among the included studies. A result higher than 50\% would represent substantial heterogeneity. If heterogeneity exits, we will conduct a subgroup analysis to examine the possible cause. ${ }^{30}$

\section{Assessment of reporting biases}

If more than 10 trials are included in the meta-analysis, we will conduct funnel plots to assess the potential for publication bias and small study effects. ${ }^{31}$ Asymmetry in funnel plots implied possible small study effects, such as publication bias. We will include all eligible trials, regardless of their methodological quality.

\section{Data synthesis}

If there are sufficient studies and comparable outcomes, we will perform a meta-analysis using random effect modelling.

1. Herbal medicine versus conventional medical treatments

2. Herbal medicine versus no treatment

3. Herbal medicine versus placebo

4. Herbal medicine plus conventional medical treatment versus conventional medical treatment only

\section{Subgroup analysis and investigation of heterogeneity}

If there are sufficient data, subgroup analyses will be conducted to explore the differences in the effect sizes, type of cancer, stage of cachexia ${ }^{4}$ and types of herbal medicine.

\section{Sensitivity analysis}

For sensitivity analysis, the meta-analysis will be repeated, substituting decisions alternatively to test the robustness of the primary decisions of the review process. The principal decision nodes are as follows:

1. Methodological quality (sequence generation, allocation concealment or blinding in the assessment of outcomes and symptom severity);

2. Sample size (small sample size studies, eg, over 30 in each group). 


\section{ETHICS AND DISSEMINATION}

Ethical approval is not required, given that this protocol is for a systematic review. The review will be disseminated widely through peer-reviewed publications and conference presentations.

\section{DISCUSSION}

This is the protocol for a review and there is no primary data collection. The systematic review will be published in a peer-reviewed journal and disseminated electronically or in print. Updates of the review will be conducted to inform and guide healthcare practice and policy.

Contributors The search strategy was developed and will be run by BP and JHJ. Copies of studies will be obtained by BP and JHJ. Selection of the studies to include will be performed by BP and JHJ. MSL will act as an arbiter in the study selection stage. Extraction of data from studies will be conducted by BP, JHJ, JJ and SY. Entering data into RevMan will be conducted by BP and JJ. The analysis will be carried out by BP, JJ and MSL. Interpretation of the analysis will be performed by all authors. The final review will be drafted by all authors. The review will be updated by BP and MSL. All authors (BP, JHJ, JJ, SY and MSL) contributed to the drafting of the protocol. The protocol was revised, and the final version was approved by all authors.

Funding The authors were supported by the Korea Institute of Oriental Medicine (K13400, K13281).

Competing interests None.

Provenance and peer review Not commissioned; externally peer reviewed.

Open Access This is an Open Access article distributed in accordance with the Creative Commons Attribution Non Commercial (CC BY-NC 3.0) license, which permits others to distribute, remix, adapt, build upon this work noncommercially, and license their derivative works on different terms, provided the original work is properly cited and the use is non-commercial. See: http:// creativecommons.org/licenses/by-nc/3.0/

\section{REFERENCES}

1. Tuca A, Jimenez-Fonseca P, Gascon P. Clinical evaluation and optimal management of cancer cachexia. Crit Rev Oncol Hematol 2013;88:625-36.

2. Evans WJ, Morley JE, Argiles J, et al. Cachexia: a new definition. Clin Nutr 2008;27:793-9.

3. Muscaritoli M, Anker SD, Argiles J, et al. Consensus definition of sarcopenia, cachexia and pre-cachexia: joint document elaborated by Special Interest Groups (SIG) "cachexia-anorexia in chronic wasting diseases" and "nutrition in geriatrics". Clin Nutr 2010;29:154-9.

4. Fearon K, Strasser F, Anker SD, et al. Definition and classification of cancer cachexia: an international consensus. Lancet Oncol 2011:12:489-95.

5. Douglas E, McMillan DC. Towards a simple objective framework for the investigation and treatment of cancer cachexia: the Glasgow Prognostic Score. Cancer treatment reviews. Cancer Treat Rev 2014;40:685-91.

6. Tisdale MJ. Mechanisms of cancer cachexia. Physiol Rev 2009;89:381-410

7. Tsoli M, Robertson G. Cancer cachexia: malignant inflammation, tumorkines, and metabolic mayhem. Trends Endocrinol Metab 2013;24:174-83.

8. Fearon KC, Glass DJ, Guttridge DC. Cancer cachexia: mediators, signaling, and metabolic pathways. Cell Metab 2012;16:153-66.
9. Scott HR, McMillan DC, Brown DJ, et al. A prospective study of the impact of weight loss and the systemic inflammatory response on quality of life in patients with inoperable non-small cell lung cancer. Lung Cancer 2003;40:295-9.

10. Prado CM, Baracos VE, McCargar LJ, et al. Sarcopenia as a determinant of chemotherapy toxicity and time to tumor progression in metastatic breast cancer patients receiving capecitabine treatment. Clin Cancer Res 2009;15:2920-6.

11. Ruiz Garcia V, Lopez-Briz E, Carbonell Sanchis R, et al. Megestrol acetate for treatment of anorexia-cachexia syndrome. Cochrane Database Syst Rev 2013;3:CD004310.

12. Argiles JM, Olivan M, Busquets $\mathrm{S}$, et al. Optimal management of cancer anorexia-cachexia syndrome. Cancer Manag Res 2010;2:27-38

13. Nicolini A, Ferrari P, Masoni MC, et al. Malnutrition, anorexia and cachexia in cancer patients: a mini-review on pathogenesis and treatment. Biomed Pharmacother 2013:67:807-17.

14. Lawsin C, DuHamel K, Itzkowitz SH, et al. Demographic, medical, and psychosocial correlates to CAM use among survivors of colorectal cancer. Support Care Cancer 2007:15:557-64.

15. Matthews AK, Sellergren SA, Huo D, et al. Complementary and alternative medicine use among breast cancer survivors. J Altern Complement Med 2007;13:555-62.

16. Kang S, Min H. Ginseng, the 'Immunity Boost': The Effects of Panax ginseng on Immune System. J Ginseng Res 2012;36:354-68.

17. Shergis JL, Zhang AL, Zhou W, et al. Panax ginseng in randomised controlled trials: a systematic review. Phytother Res 2013;27:949-65.

18. Harrold JA, Hughes GM, O'Shiel K, et al. Acute effects of a herb extract formulation and inulin fibre on appetite, energy intake and food choice. Appetite 2013;62:84-90.

19. Ren S, Zhang H, Mu Y, et al. Pharmacological effects of astragaloside IV: a literature review. J Tradit Chin Med 2013;33:413-16.

20. Lee JJ, Lee JJ. A phase II study of an herbal decoction that includes astragali radix for cancer-associated anorexia in patients with advanced cancer. Integr Cancer Ther 2010;9:24-31.

21. Lou $T$, Zhang Z, Xi Z, et al. Berberine inhibits inflammatory response and ameliorates insulin resistance in hepatocytes. Inflammation 2011;34:659-67.

22. Zhang J, Wang $\mathrm{P}$, Ouyang $\mathrm{H}$, et al. Targeting cancer-related inflammation: Chinese herbal medicine inhibits epithelial-tomesenchymal transition in pancreatic cancer. PLOS ONE 2013;8: e70334.

23. He DY, Dai SM. Anti-inflammatory and immunomodulatory effects of paeonia lactiflora pall., a traditional chinese herbal medicine. Front Pharmacol 2011;2:10

24. Wang S, Wu X, Tan M, et al. Fighting fire with fire: poisonous Chinese herbal medicine for cancer therapy. $J$ Ethnopharmacol 2012;140:33-45.

25. Lu JJ, Dang YY, Huang M, et al. Anti-cancer properties of terpenoids isolated from rhizoma curcumae-a review. $J$ Ethnopharmacol 2012;143:406-11.

26. Chen X, Guo J, Bao J, et al. The anticancer properties of salvia miltiorrhiza bunge (Danshen): a systematic review. Med Res Rev Published Online First: 7 October 2013. doi: 10.1002/med.21304

27. Takeda H, Muto S, Nakagawa K, et al. Rikkunshito as a ghrelin enhancer. Methods Enzymol 2012;514:333-51.

28. lizuka N, Miyamoto K, Hazama S, et al. Anticachectic effects of Coptidis rhizoma, an anti-inflammatory herb, on esophageal cancer cells that produce interleukin 6. Cancer Lett 2000;158: $35-41$.

29. Higgins JPT, Altman DG, Sterne JAC. Chapter 8: assessing risk of bias in included studies. In: Higgins JPT, Green S. eds Cochrane handbook for systematic reviews of interventions version 5.1.0 (updated March 2011). The Cochrane Collaboration. http://www. cochrane-handbook.org, 2011.

30. Deeks JJ, Higgins JPT, Altman DG. Chapter 9: analysing data and undertaking meta-analyses. In: Higgins JPT, Green S. eds Cochrane handbook for systematic reviews of interventions version 5.1.0 (updated March 2011). The Cochrane Collaboration. 2011. http:// www.cochrane-handbook.org

31. Egger M, Davey Smith G, Schneider M, et al. Bias in meta-analysis detected by a simple, graphical test. BMJ 1997;315:629-34. 